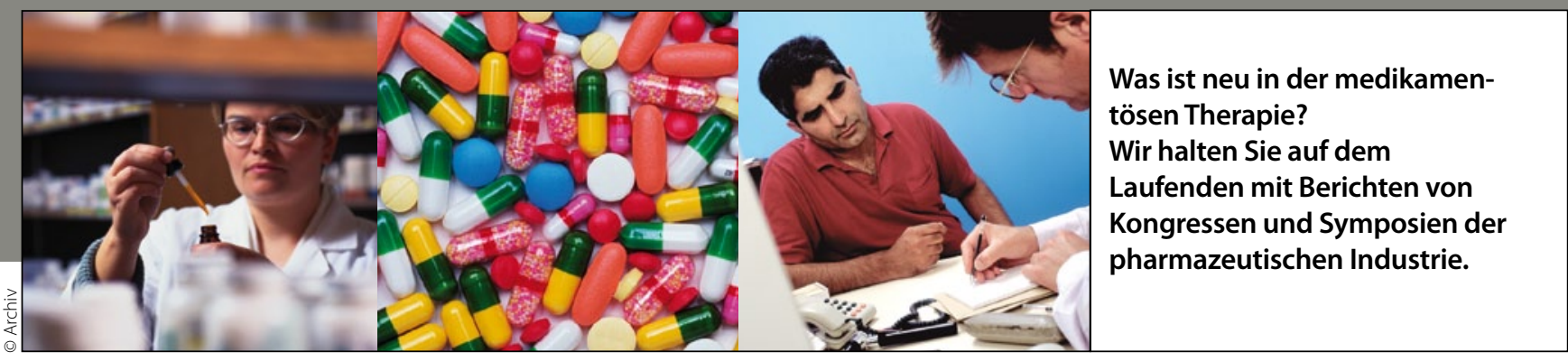

Direkter Faktor-Xa-Hemmer Rivaroxaban

\section{Therapeutisches Potenzial noch nicht ausgeschöpft?}

- Das therapeutische Potenzial von Rivaroxaban ist wohl selbst nach der jüngst erfolgten Zulassungserweiterung zur Therapie und Sekundärprophylaxe der Lungenembolie noch nicht ausgeschöpft. Zur Zeit läuft ein Zulassungsantrag zur Sekundär-Prävention bei Patienten nach akutem Koronarsyndrom.

Die Antikoagulation blickt auf eine Erfolgsgeschichte zurück, die in den 1930er Jahren mit unfraktioniertem Heparin und den Vitamin-K-Antagonisten begann und zur Entwicklung der modernen Antikoagulanzien führte. Inzwischen sind die modernen Wirkstoffe in verschiedenen Indikationen zugelassen, wobei der direkte Faktor-Xa-Hemmer Rivaroxaban das breiteste Indikationsspektrum besitzt. Es reicht von der Prophylaxe venöser Throm- boembolien nach elektiver Knie- und Hüftgelenksersatzoperation über die Schlaganfallprophylaxe bei Vorhofflimmern und die Behandlung und Sekundärhin zur Behandlung und Sekundärprophylaxe von Lungenembolien.

\section{Therapie und Sekundärprophylaxe der Lungenembolie}

In dieser Indikation gab es für Rivaroxaban jüngst eine Zulassungserweiterung, nachdem in der EINSTEIN-PE-Studie bei mehr als 4800 Patienten gezeigt wurde, dass die Substanz ebenso wirksam ist wie die bisherige Standardtherapie, allerdings zu signifikant weniger schweren Blutungen führt. So betrug die Inzidenzrate schwerer Blutungen lediglich 1,1\% gegenüber prophylaxe tiefer Venenthrombosen bis

\section{Abbildung 1}

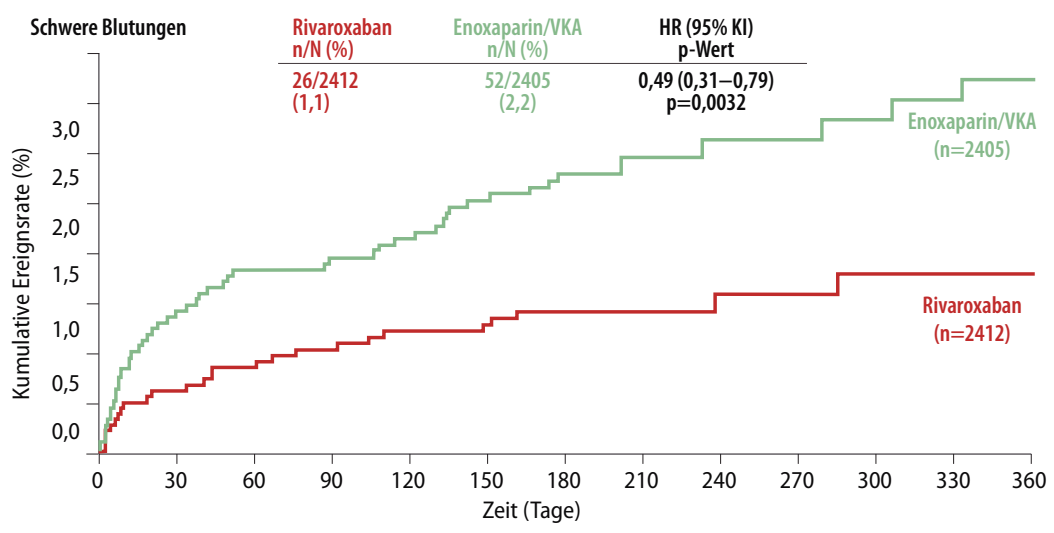

Abb. 1 Unter Rivaroxaban kam es zu signifikant weniger schweren Blutungen (sekundärer Sicherheitsendunkt der Einstein-PE-Studie).

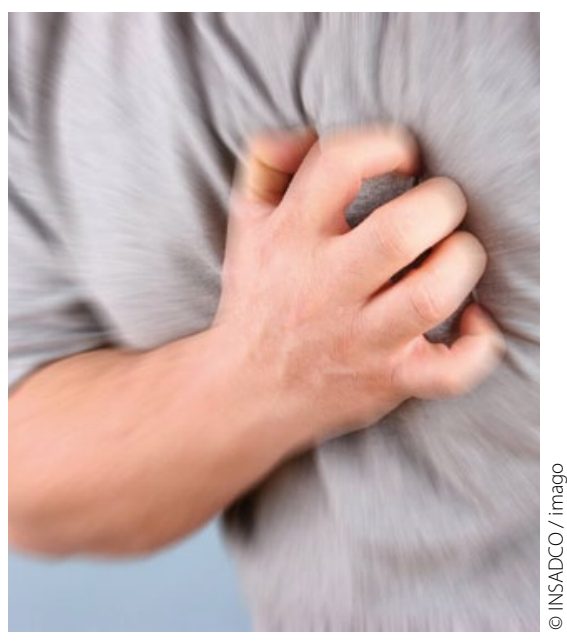

Das akute Koronarsyndrom - bald auch eine Indikation für den Einsatz von Rivaroxaban?

2,2\% unter der Standardtherapie (Abb. 1), berichtete Dr. Frank Misselwitz, Wuppertal. Ein weiterer Vorteil bestehe darin, dass mit nur einem einzigen Wirkstoff Therapie wie auch Sekundärprophylaxe zu realisieren seien, sagte der Mediziner, der maßgeblich an der Entwicklung von Rivaroxaban beteiligt war.

\section{Zulassungsantrag für dem Einsatz beim akuten Koronarsyndrom}

Zu erwarten ist aus seiner Sicht eine weitere Zulassung für den Faktor-Xa-Hemmer, da dieser auch bei der Sekundärprävention des akuten Koronarsyndroms bereits seine klinische Wirksamkeit und Sicherheit in Studien unter Beweis gestellt hat.

- Christine Vetter

Quelle: Pressegespräch „AntithromboseForschung auf der Höhe der Zeit" anlässlich der 57. Jahrestagung der Gesellschaft für Thrombose- und Hämostaseforschung (GTH), München, Februar 2013 (Veranstalter: Bayer Healthcare) 